\title{
АНАЛИЗ СИСТЕМЫ МАТЕМАТИЧЕСКОГО ОБЕСПЕЧЕНИЯ ТРЕНАЖЕРНОГО ЧЕЛОВЕКО-МАШИННОГО КОМПЛЕКСА ПОЛУНАТУРНОГО МОДЕЛИРОВАНИЯ СИСТЕМЫ УПРАВЛЕНИЯ ЛЕТАТЕЛЬНЫМ АППАРАТОМ
}

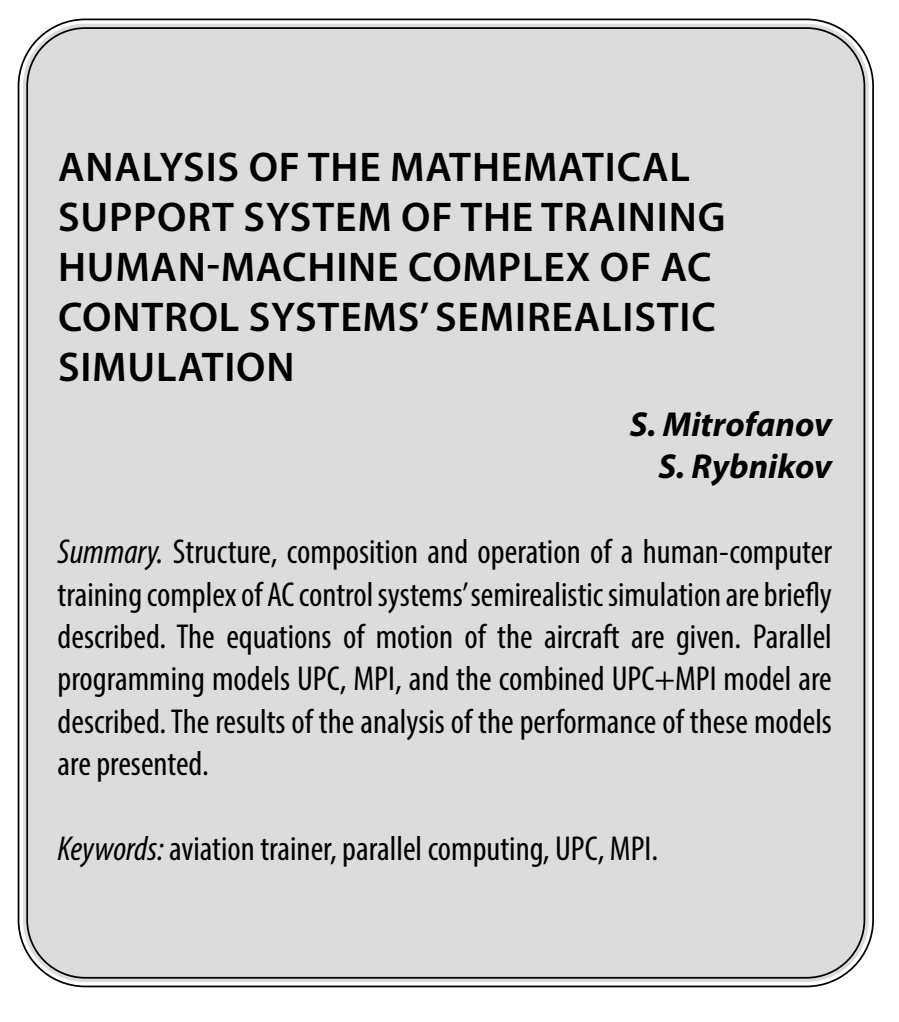

\section{Введение}

A виационный тренажер (АТ) представляет собой технически сложную систему, использующую технологии моделирования для разработки и тестирования систем авионики самолета, а также безопасной подготовки и переподготовки авиационных специалистов различных категорий. Ключевым критерием эффективности АТ является возможность использования приобретаемых во время обучения навыков «на земле». На эффективность обучения летного персонала влияет множество факторов, среди которых особую роль выполняет степень достоверности воспроизведения в процессе тренировок всего комплекса воспринимаемых ощущений и потоков информации в сравнении с аналогичной ситуацией в полете на реальном воздушном судне. Такая достоверность достигается не только использованием блоков авионики с ЛА, но и построением правильной архитектуры взаимодействия систем тренажера и обмена информации между ними.
Митрофанов Сергей Юрьевич

Aспирант, ФГБОУ ВО «Московский авиационный институт (национальный исследовательский университет)» seymitrofanov@mai.ru

Рыбников Сергей Игоревич

Д.т.н., ФГБОУ ВО «Московский авиачионный институт (национальный исследовательский университет)»

Аннотация. Кратко описывается состав, структура, работа тренажерного человеко-машинного комплекса полунатурного моделирования СУ ЛА. Приводятся дифференциальные уравнения движения ЛА для построения математической модели воздушного судна. Описываются модели параллельного программирования UPC, MPI и объединенная модель UPC+MPI. Приводятся результаты анализа производительности данных моделей.

Ключевые слова: авиационный тренажер, параллельное программирование, UPC, MPI.

\section{Структура авиационного тренажера}

Комплексный пилотажный тренажер представляет собой полноразмерную модель кабины экипажа самолета определенного типа или марки, модели и серии, включающая полный набор оборудования и компьютерных программ, необходимый для имитации функционирования самолета на земле и в полете, систему визуализации, имитирующую внешний обзор из кабины экипажа, и систему координирования движущих приводов [1] (Рисунок 1).

Структура АТ, в соответствии с нормативными документами [2,3], представлена на рисунке 2.

Уравнения движения моделируемого самолета являются основой АТ. Силы и моменты, определяющие движение самолета, решаются как набор дифференциальных уравнений для расчета движения и траектории самолета. Динамические уравнения движения центра 


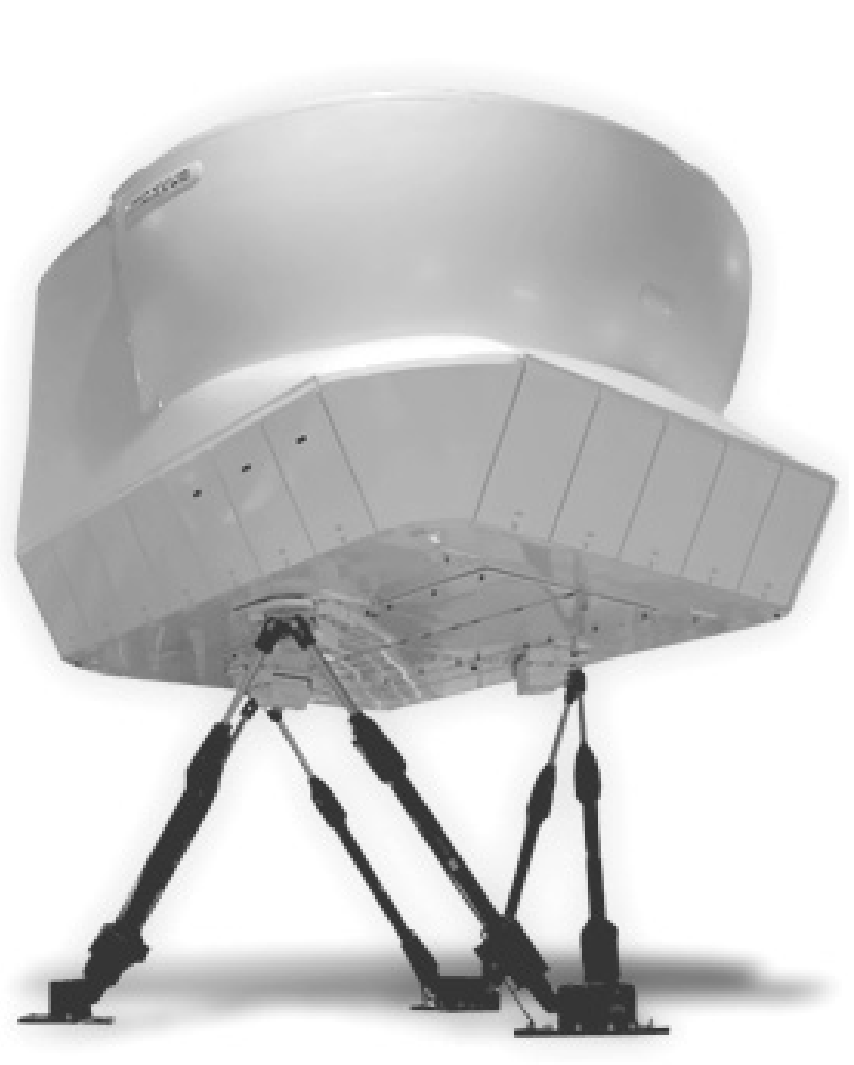

Рис. 1. Комплексный пилотажный тренажер

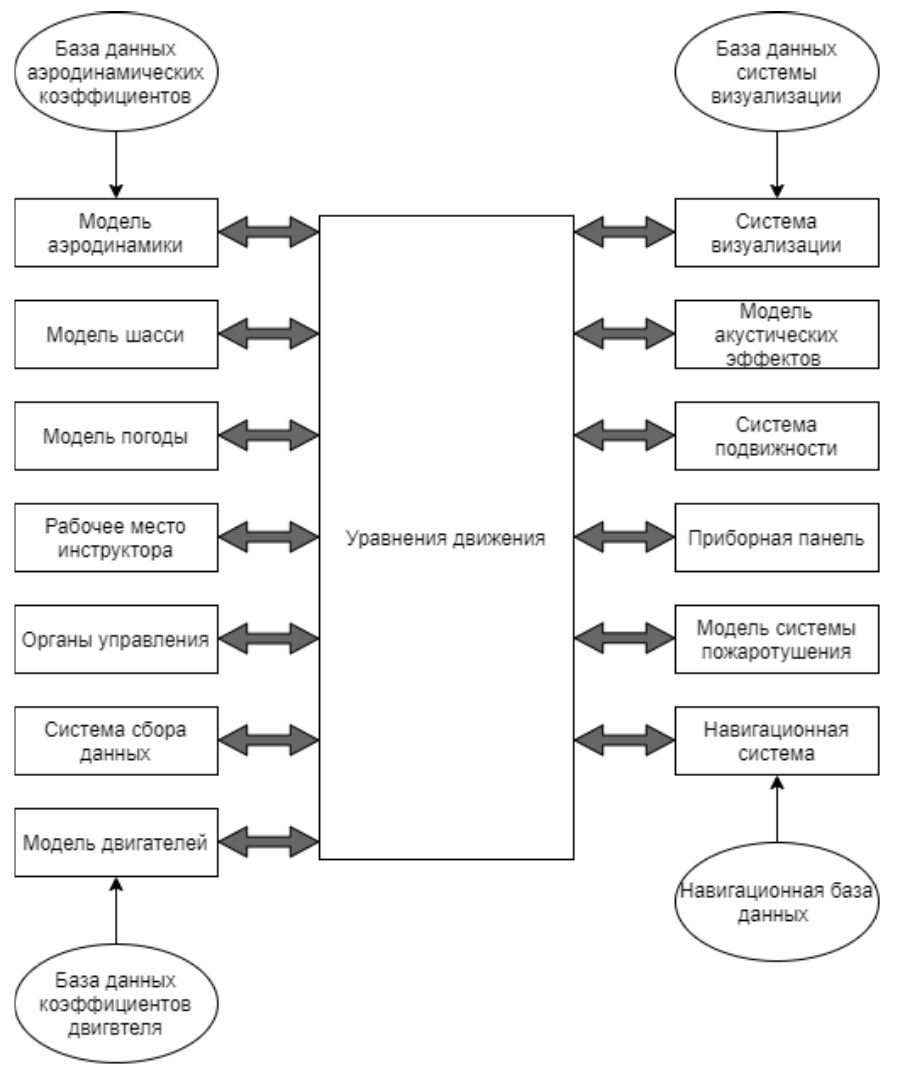

Рис. 2. Структура авиационного тренажера

$$
\begin{aligned}
& m\left(\frac{d V_{k x}}{t d}+\omega_{y} V_{k z}-\omega_{z} V_{k y}\right)=F_{x} \\
& m\left(\frac{d V_{k y}}{t d}+\omega_{z} V_{k x}-\omega_{x} V_{k z}\right)=F_{y} \\
& m\left(\frac{d V_{k z}}{t d}+\omega_{x} V_{k y}-\omega_{y} V_{k x}\right)=F_{z} \\
& J_{x} \dot{\omega}_{x}+\left(J_{z}-J_{y}\right) \omega_{y} \omega_{z}-J_{x y}\left(\dot{\omega}_{y}-\omega_{x} \omega_{z}\right)-J_{x z}\left(\dot{\omega}_{z}+\omega_{x} \omega_{y}\right)+J_{y z}\left(\omega_{z}^{2}-\omega_{y}^{2}\right)=M_{x} \\
& J_{y} \dot{\omega}_{y}+\left(J_{x}-J_{z}\right) \omega_{z} \omega_{x}-J_{y z}\left(\dot{\omega}_{z}-\omega_{y} \omega_{x}\right)-J_{x y}\left(\dot{\omega}_{x}+\omega_{x} \omega_{z}\right)+J_{z x}\left(\omega_{x}^{2}-\omega_{z}^{2}\right)=M_{y} \\
& J_{z} \dot{\omega}_{z}+\left(J_{y}-J_{x}\right) \omega_{x} \omega_{y}-J_{z x}\left(\dot{\omega}_{x}-\omega_{z} \omega_{y}\right)-J_{z y}\left(\dot{\omega}_{y}+\omega_{z} \omega_{x}\right)+J_{x y}\left(\omega_{y}^{2}-\omega_{x}^{2}\right)=M_{z} \\
& \dot{\psi}=\frac{1}{\cos \vartheta}\left(\omega_{y} \cos \gamma-\omega_{z} \sin \gamma\right) \\
& \quad \dot{\vartheta}=\omega_{y} \sin \gamma+\omega_{z} \cos \gamma \\
& \dot{\gamma}=\omega_{x}-\tan \vartheta\left(\omega_{y} \cos \gamma-\omega_{z} \sin \gamma\right)
\end{aligned}
$$

масс в проекциях на оси связанной системы координат имеют вид [4]:

Здесь $F_{x}, F_{y}, F_{z}$ - проекции суммарного вектора внешних сил на продольную, нормальную и поперечную оси; $w_{x}, w_{y}, w_{z}$ - проекции угловой скорости; $V_{k x}, V_{k y}, V_{k z}-$ проекции путевой скорости; $m$ - масса самолета.
Динамические уравнения движения относительно центра масс в проекциях на оси связанной системы координат имеют вид [4]:

Здесь $M_{x}, M_{y}, M_{z}$ - проекции суммарного момента внешних сил на продольную, нормальную и поперечные оси; $J_{x \prime} J_{y \prime} J_{z \prime} J_{x y}, J_{x z}, J_{y z}-$ моменты инерции. 
Процесс

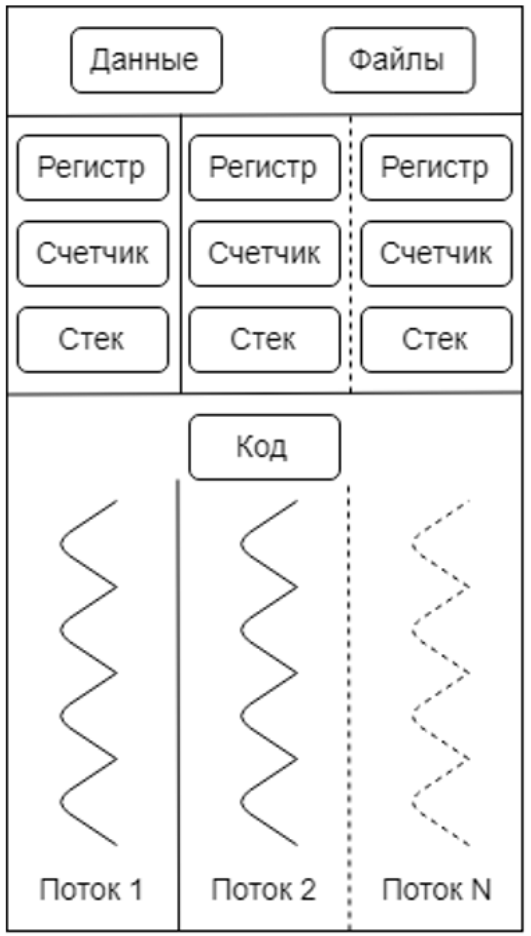

Рис. 3. Многопоточный процесс

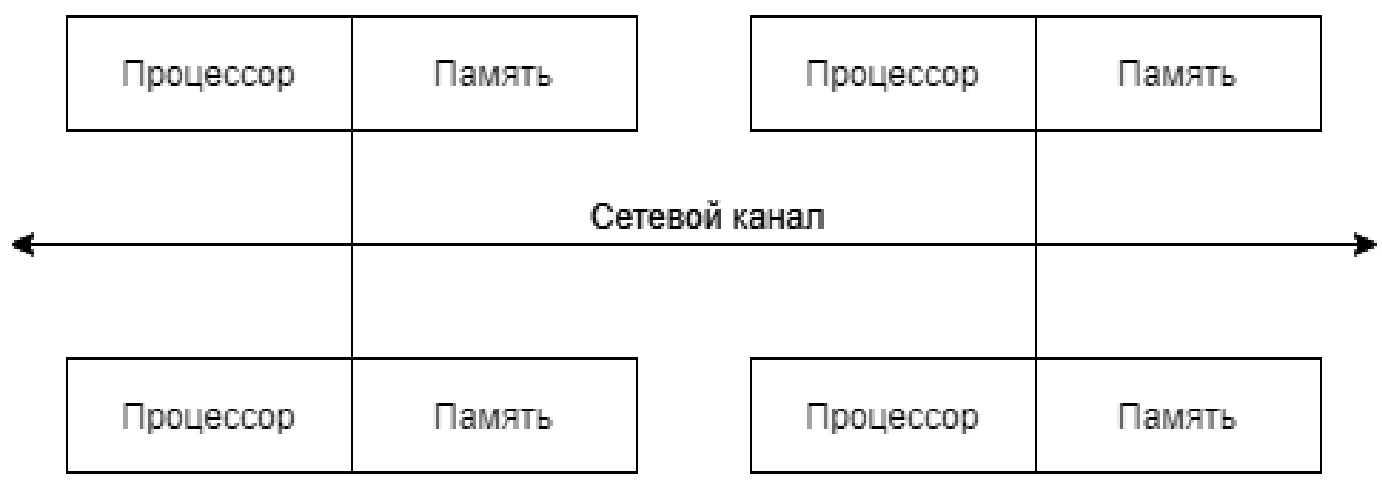

Рис. 4. Классическая архитектура модели MPI

Для описания кинематики вращательного движения ЛА в АТ применяют кинематические уравнения Эйлера [5]:

где $\psi, \vartheta, ~ ү-$ - углы рысканья, тангажа и крена.

Моделирование проводится с помощью соответствующего программного обеспечения на персональных компьютерах с необходимой точностью, для адекватного воспроизведения движения самолета. Кроме того, моделирование должно происходить в реальном времени. Следовательно, разработке архитектуры ПО АТ необходимо уделять особое внимание.

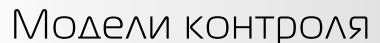 \\ распреАеления $\triangle$ анных}

\section{Модель Unified Parallel C}

Unified Parallel C (UPC) - это явный параллельный язык, предоставляющий возможности для непосредственной спецификации пользователем параллелизма программ и управления распределением и доступом к данным. Разработчику ПО предоставляется доступ к единому и совместно используемому глобальному адресному пространству [6]. Количество потоков (Рисунок 3) или степень параллелизма определяется во время 
UPC Группа

математической модели самолета

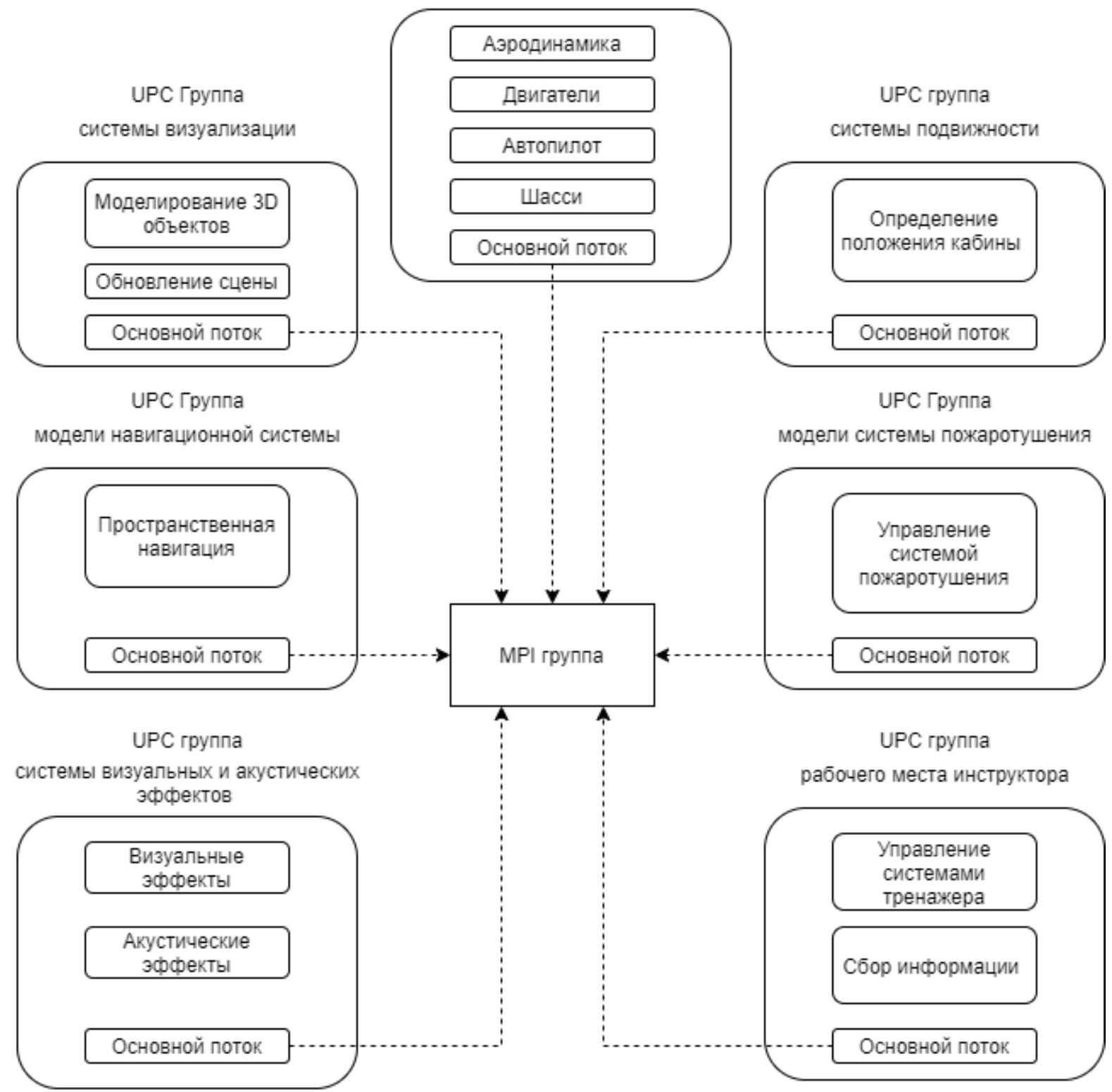

Рис. 5. Архитектура совмещенной модели UPC и MPI для АT

запуска компилятора, либо во время запуска программы и не изменяется во время выполнения.

Каждый из запущенных потоков выполняет одну и ту же программу, однако работают они независимо и имеют личное адресное пространство. UPC позволяет оптимизировать распределение данных и координировать взаимодействие потоков таким образом, что время выполнения операций уменьшается в сравнении с последовательными вычислениями. Модель UPC позволяет частично разделять на потоки последовательные вы- числительные процессы, что способствует увеличению общей производительности ПО.

\section{Модель Message Passing Interface}

Message Passing Interface (MPI) - широко распространенный программный интерфейс передачи информации, позволяющий организовать обмен сообщениями между процессами, выполняющими единую задачу. Модель МРI часто используется при разработке ПО для параллельных компьютеров с распределенной памятью 

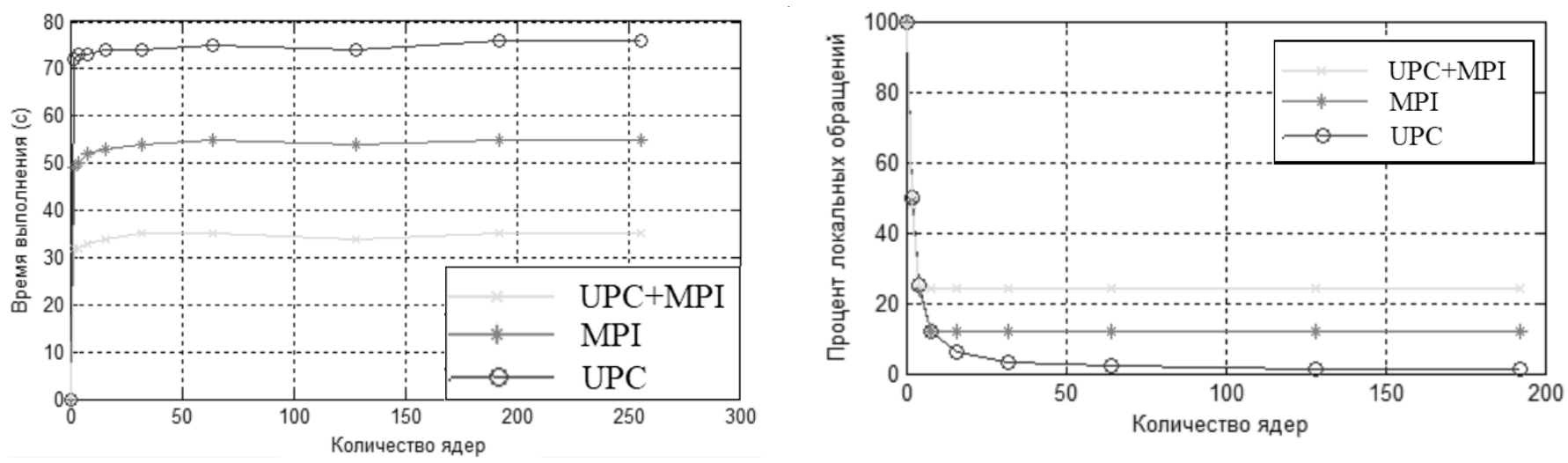

Рис. 6. Результаты анализа моделей

и объединенных высокоскоростными каналами связи (Рисунок 4). Стандарты MPI поддерживают как двусторонние операции отправки/получения сообщений во время обмена данными между парами процессов, так и одностороннюю связь.

\section{Коншепция совмешенной модели UPC и MPI}

Модели UPC и MPI по отдельности имеют свои преимущества и недостатки, замедляющие скорость обработки информации и работу ПО. Оптимальная модель MPI требует достаточной детализации сообщений для обмена данными, что является весьма энергозатратным действием даже для современных высокопроизводительных ПК. Совмещенная модель предполагает использование UPC для организации внутренних параллельных потоков, а MPI внешних.

При объединении моделей UPC и MPI предлагается определить группы UPC, связанные между собой только обменом данных с помощью MPІ. Поэтому, при разработке такой системы, необходимо определить количество запускаемых групп и принять решение о разделении потоков в каждой группе. Один из потоков выбирается в качестве главного для обслуживания процессов МPI и UPC.

\section{Применение совмещенной модели UPC и MPI в АT}

Предлагаемая архитектура совмещенной модели UPC и MPI для использования в комплексном пилотажном тренажере изображена на рисунке 5. Такая архитектура позволит организовать взаимодействие между системами АT. Группы UPC разделяются по основным моделям: математическая модель самолета, система подвижности, система визуализации, модель навигационной системы, рабочее место инструктора, модель системы пожаротушения, система визуальных и акустических эффектов.
Основные потоки групп объединяются в группу MPI для организации обмена сообщениями между ними.

\section{Анализ произволительности}

Анализ производительности - запуск программы тестирования для анализа производительности системы, какой-то её части или непосредственно программы. Анализ необходим и важен для понимания вычислительных ресурсов на различных платформах, так как невозможно произвести достоверную оценку исходя из данных, представленных в технических характеристиках. В данном случае, при использовании параллельных программ в тренажерном устройстве имитации полета такая информация необходима для обеспечения адекватности AT.

На основе методов, описанных в [7], были проведены тесты для моделей UPC и MPI отдельно и для совмещенной модели UPC и MPI. Результаты представлены на рисунке 6.

В данной системе использовались шестиядерные процессоры Intel Core i5 с тактовой частотой 2.8 Ггц. У каждого процесса есть постоянный объем выполняемых операций, поэтому производительность сохраняется при увеличении количества процессоров. Исходя из представленных результатов, можно сделать вывод, что совмещенная модель UPC и MPI превосходит по производительности модель UPC примерно в 2 раза и в 1.5 раза по сравнению с моделью МРI.

\section{Зак^ючение}

В настоящее время авиационные тренажеры являются неотъемлемой частью программ профессиональной подготовки летных экипажей различных категорий. Для качественной подготовки авиационных специалистов тренажеры должны соответствовать 
критериям эффективности обучающих средств, важным фактором которых является степень достоверности воспроизведения воспринимаемых обучающимся ощущений и потоков информации. Уравнения (1), (2), (3) - базовые уравнения математической модели самолета в АT.
Предлагается архитектура модели параллельного программирования для организации взаимодействия основных систем тренажера. Проведенный анализ моделей UPC, MPI и совмещенной UPC+MPI показывает эффективность модели UPC+MPI в сравнении с базовыми за счет роста производительности.

1. David Allerton, Principles of Flight Simulation, John Wiley \& Sons, Ltd, 2009

2. CS-FSTD(A) «Certification Specifications for Aeroplane Flight Simulation Training Devices».

3. Manual of Criteria for the Qualification of Flight Simulation Training Devices - Volume II - Aeroplanes (9625-2)

4. Динамика полета: Учебник для авиационных вузов / Мхитрян А. М., Лазнюк П. С., Максимов В. С. и др.— М.: Машиностроение, 1978. - 424с.

5. Пашковский И. М. Динамика и управляемость самолета.— М.: Машиностроение, 1987.— 429 с.

6. UPC: Distributed Shared Memory Programming (Wiley Series on Parallel and Distributed Computing Book 73) 1st Edition, Kindle Edition, 2007

7. Н.Е. Андреев, К.Е. Афанасьев, Реализация инструментального средства автоматизированного анализа производительности UPC-программ, Вычислительные методы и программирование, 2011, том 12, выпуск 2, 46-57

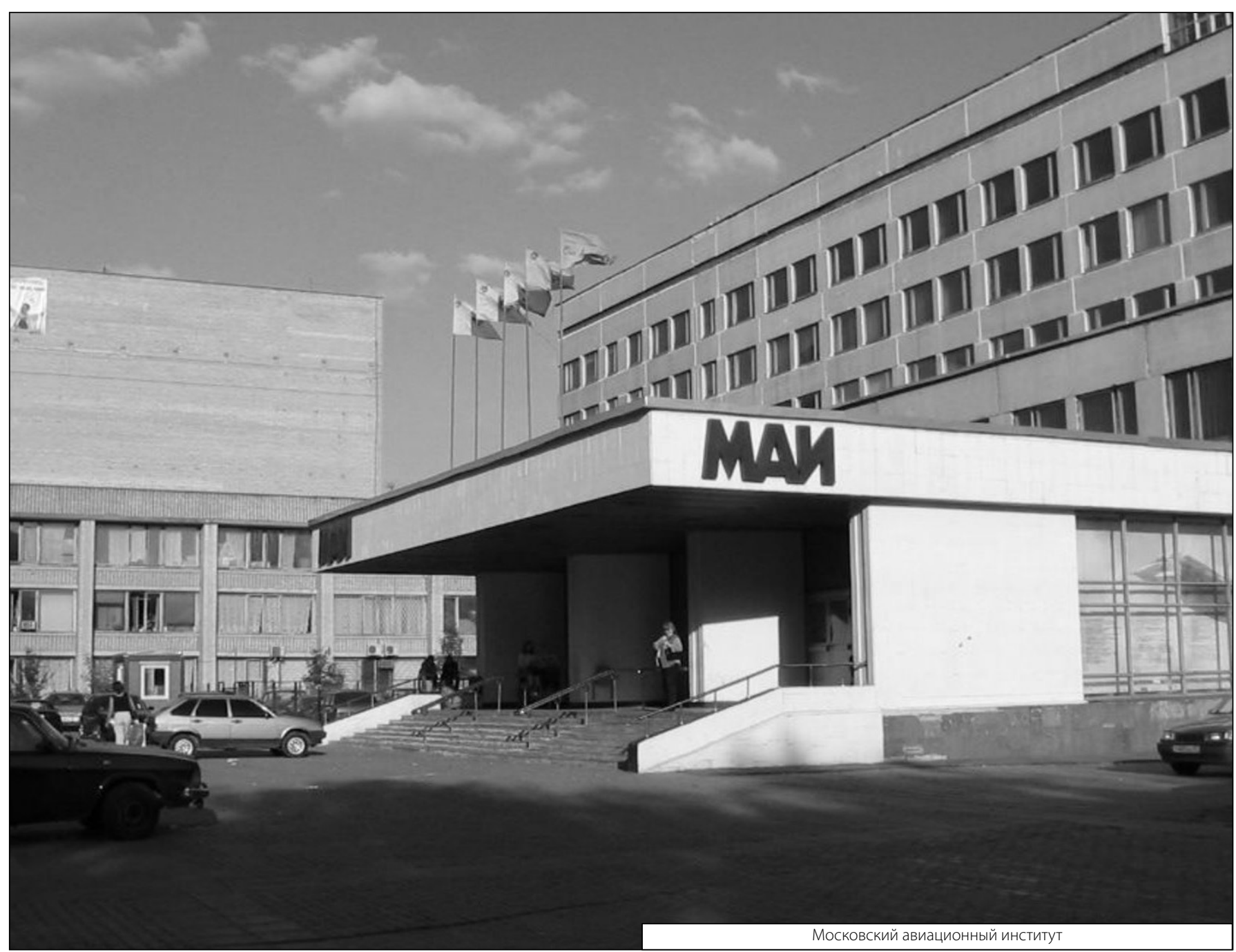

